\title{
ANÁLISES FÍSICO-QUÍMICAS DE AMOSTRAS DE MÉIS DE FLORES SILVESTRES, DE EUCALIPTO E DE LARANJEIRA, PRODUZIDOS POR Apis mellifera NO ESTADO DE SÃO PAULO. 1. ÍNDICE DE DIASTASE E HIDROXIMETILFURFURAL ${ }^{1}$.
}

Sônia Shigueyo Komatsu Luís Carlos Marchini Augusta Carolina de Camargo Carmello Moreti $^{3}$ RESUMO

Em 94 amostras de méis de flores silvestres, 27 de flores de eucalipto e 34 de flores de laranjeira (totalizando 155 amostras) produzidas por Apis mellifera L., 1758 (Hymenoptera, Apidae), em 96 municípios do Estado de São Paulo foram determinados o índice de diástase, o hidroximetilfural (HMF) e a porcentagem de amostras que se enquadram nas especificações da legislação brasileira. De acordo com os dados obtidos, conclui-se que a caracterização das amostras estudadas no presente trabalho é estabelecida por faixas de variação de valores obtidos de 5,0 a 38,5 para o índice de diastase e 0,3 a $242,6 \mathrm{mg} / \mathrm{kg}$ para HMF. Das amostras analisadas $93,6 \%$ se enquadram nas especificações da legislação brasileira para qualidade de mel para índice de diástase e $94,8 \%$ para HMF.

Palavras-chave: mel, Apis mellifera, análises físico-químicas, diástase, HMF

1 Parte da tese de Doutorado defendida pela primeira autora em 03/04/1996, ESALQ/USP. 2 Dep. Entomologia, Fitopatologia e Zoologia Agrícola, ESALQ/USP, Piracicaba, SP. 3 Centro de Etologia, Ambiência e Manejo, Instituto de Zootecnia, Nova Odessa, SP. Bolsista do CNPq. 


\section{ABSTRACT}

\section{PHYSICO-CHEMICAL ANALYSIS OF SAMPLES OF HONEY FROM WILD FLOWERS, Eucalyptus FLOWERS AND Citrus FLOWERS PRODUCED BY Apis mellifera IN THE STATE OF SÃO PAULO, BRAZIL. 1. DIASTASE NUMBER AND HIDROXYMETHYLFURFURAL}

A total of 155 honey samples, being 94 from wild flowers, 27 from Eucalyptus flowers and 34 from Citrus flowers produced by Apis mellifera were collected from 96 locations in the State of São Paulo. The following parameters were analysed: the diastase values, hydroxymethylfurfural and the percentage of honey samples that followed the Brazilian legislation for honey quality. According to the results, the characterization of honeys was established by ranges for each parameter studied: 5.0 to 38.5 for diastase and 0.3 to $242.6 \mathrm{mg} / \mathrm{kg}$ for HMF. The percentage of honey samples according to the Brazilian legislation for honey quality, for each parameter evaluated were: $93.6 \%$ for diastase value and $94.8 \%$ for HMF.

Key words: honey, Apis mellifera, physico-chemical analysis, diastase values, $\mathrm{HMF}$.

\section{INTRODUÇÃo}

O mel, um produto genuinamente natural, há milênios vem sendo utilizado como alimento pela humanidade. Além de seu valor nutritivo, apresenta algumas características terapêuticas, sendo, ainda, muito utilizado pela indústria alimentícia e de cosméticos. No Brasil, onde o mel não é consumido rotineiramente, por falta de hábito, não existe um padrão que expresse sua qualidade baseando-se em características químicas e físicas. Por esse motivo, até hoje, são seguidos, em nosso país, os padrões estabelecidos pelos norte-americanos.

A flora apícola brasileira é bastante diversificada, variando de 
egião para região e por isso, torna-se necessária à caracterização e poserior criação de padrões para tais méis, de acordo com os fatores vegetais, ¿dáficos e climáticos das respectivas regiões em que foram produzidos.

As mais extensas áreas apícolas correspondem à vegetação silvestre e sua diversidade de espécies botânicas torna disponível às abethas, durante o ano todo, um grande variedade de flores. Os pomares cítricos, por sua vez, com suas breves floradas, fornecem matéria prima para a elaboração de méis muito apreciados pelos consumidores e, ainda, as reservas florestais e áreas de reflorestamento com eucalipto, consistem num farto pasto apícola. Diante do exposto, essas três fontes de alimento são as principais produtoras do mel encontrado no comércio brasileiro.

O índice de diástase e o conteúdo de hifroximetilfurfural são dois dos parâmetros rotineiramente determinados nas análises de mel e indicam não só a idade, mas também um eventual superaquecimento do produto. Segundo Vansell \& Freedon (1929) existe uma perfeita correlação entre a quantidade de pólen presente no mel e a atividade da diastase.

Persono-Oddo et al. (1990) estudaram a presença de enzimas de méis uniflorais italianos de diferentes origens botânicas com o objetivo de apresentar a variação no valor da diastase. Concluíram que essa variabilidade se deve, provavelmente, a uma série de fatores extrínsicos e intrínsicos incontroláveis.

White Júnior (1992) afirmou que méis de regiões quentes e secas apresentam menor quantidade de enzimas se comparados com os de regiões frias e úmidas.

Azeredo \& Azeredo (1999) avaliaram amostras de méis de São Fidélis, RJ, armazenadas em diferentes condições e constataram que com o passar do tempo, independentemente do tipo de recipiente e da forma de armazenamento, ocorreu diminuição no índice de diastase.

O Hidroximetilfurfural (HMF) é o produto formado por meio do aquecimento ou armazenamento do mel. É um parâmetro que, além do superaquecimento, também pode indicar a idade do mel (Salinas et al., 1991). Segundo Veríssimo (1988) o HMF é um indicador de qualidade do mel, visto que, quando seu valor é elevado, indica uma queda consi- 
derável no valor nutritivo do produto, pela destruição, por meio do aquecimento, de algumas vitaminas e enzimas que são termolábeis.

White Júnior (1978) cita que o HMF é formado pela reação de certos açúcares com ácidos. O HMF presente no mel pode aumentar com a elevação da temperatura, sendo ainda afetado pela acidez, o pH, o conteúdo de água e minerais do mel. Seemann \& Neira (1988) relataram que o HMF se forma por decomposição da frutose em presença de ácidos. Segundo esses autores, além do aquecimento e armazenamento, o HMF é formado por adição de açúcar invertido.

Diante da escassez de informações sobre as características físicoquímicas, tão necessárias ao incremento da apicultura nacional, e especialmente da paulista, o presente trabalho desenvolveu-se com o objetivo de determinar o índice de diástase e o hidroximetilfurfural de amostras de méis de diferentes origens florais, produzidos por Apis mellifera L., em municípios do Estado de São Paulo (Tabela 1), bem como, estabelecer as faixas de variação dos referidos parâmetros, comparando os resultados obtidos com as especificações da legislação brasileira.

\section{MATERIAL E MÉTODOS}

As análises físico-químicas foram realizadas no Laboratório de Apicultura do Departamento de Entomologia, Fitopatologia e Zoologia Agrícola, da ESALQ/USP, em Piracicaba, SP.

O índice de diástase foi determinado por meio de método adotado pelo Instituto de Tecnologia de Alimentos (ITAL), baseado na reação de iodo com o amido hidrolisado pela diastase. De acordo com a coloração azul observada no final de determinação, o valor do índice de diastase era obtido por meio de uma tabela do próprio método.

O HMF foi determinado de acordo com o método estabelecido pelo Laboratório de Controle de Qualidade dos Produtos Apícolas/ Instituto de Zootecnia, baseado na reação de Winkler, na qual, em meio ácido o hidroximetilfurfural, o ácido barbitúrico e a p-toluidina formam um produto de coloração vermelha, posteriormente avaliado em colorímetro fotoelétrico. 
Tabela 1. Número de amostras de méis de flores silvestres (S), de eucalipto (E) e de laranjeira (L) coletadas em diferentes municípios do Estado de São Paulo, Brasil.

\begin{tabular}{|c|c|c|c|c|c|c|c|c|}
\hline \multirow{2}{*}{\multicolumn{2}{|c|}{$\begin{array}{l}\text { Municípios do Estado de } \\
\text { Sāo Paulo }\end{array}$}} & \multicolumn{3}{|c|}{ Méis } & \multirow{2}{*}{$\begin{array}{l}\text { Municípios do Estado de } \\
\text { São Paulo }\end{array}$} & \multicolumn{3}{|c|}{ Méis } \\
\hline & & $\mathrm{S}$ & $\mathbf{E}$ & L & & $\mathrm{S}$ & E & L \\
\hline Altinópolis & & 1 & 1 & 1 & Junqueirópolis & 2 & & \\
\hline Amparo & & & 1 & & Juquitiba & 1 & & \\
\hline Andradina & & 1 & & 1 & Lagoinha & 1 & & \\
\hline Anhembi & & & 1 & & Lençóis Paulistas & 1 & 2 & \\
\hline Araçatuba & & 2 & 1 & 1 & Limeira & & & 1 \\
\hline Araraquara & & & 1 & 2 & Lins & 1 & & 1 \\
\hline Arthur Nogueira & & & & 1 & Luiz Antonio & & 2 & \\
\hline Assis & & 1 & & & Matão & & & 1 \\
\hline Auriflama & & 2 & & 1 & Mirandópolis & 1 & & \\
\hline Avaré & & & 2 & & Mirassol & 1 & & \\
\hline Bananal & & 1 & & & Mogi Mirim & & & 1 \\
\hline Barretos & & 1 & 1 & 2 & Monte Alegre do Sul & 1 & & \\
\hline Bebedouro & & & 1 & 5 & Monteiro Loubato & 1 & 1 & \\
\hline Boa Esperança do Sul & & 1 & & & Oriente & 1 & & \\
\hline Bofete & & 1 & & & Palestina & & & 1 \\
\hline Botucatu & & & 1 & & Paraibuna & 1 & & \\
\hline Brotas & & 1 & 2 & & Paranapanema & 1 & & \\
\hline Caçapava & & 1 & 1 & & Picdade & & & 1 \\
\hline Capão Bonito & & 1 & & & Piracaia & 1 & & \\
\hline Casa Branca & & & & 1 & Piracicaba & 1 & & \\
\hline Capela do Alto & & 1 & & 1 & Piracuama & 1 & & \\
\hline Castilho & & 1 & & 1 & Pirassununga & 1 & & \\
\hline Colina & & 1 & & & Presidente Prudente & 1 & & \\
\hline Conchal & & & & 2 & Redenção da Serra & 2 & & \\
\hline Corumbatai & & 1 & & & Rio Claro & 1 & 1 & \\
\hline Cunha & & 1 & & & Sabino & 2 & & \\
\hline Descalvado & & & & 1 & Santa Cruz do Rio Pardo & 1 & & \\
\hline Espírito Santo do Pinhal & & 1 & & & Santa Fé do Sul & 1 & & \\
\hline Estrela d'Oeste & & 2 & & & Santo Antonio do Pinhal & 1 & & \\
\hline Gália & & 1 & & & São Carlos & 1 & & 1 \\
\hline Getulina & & & & 1 & São João da Boa Vista & 1 & & \\
\hline Guarani d'Oeste & & 1 & & & São José do Rio Preto & 2 & 1 & 1 \\
\hline Guarei & & 2 & & & São Luís do Paraitinga & 2 & & \\
\hline Guarujá & & 1 & & & São Pedro & 2 & 1 & \\
\hline Igarapava & & 1 & & & São Roque & 3 & & \\
\hline Iha Solteira & & 1 & & & Serra Negra & 5 & & \\
\hline Ipeúna & & & 1 & & Sete Barras & 1 & & \\
\hline Itaberá & & 1 & & & Tabatinga & & & 1 \\
\hline Itanhaém & & 1 & & & Taquaritinga & & & 1 \\
\hline Itapetininga & & 1 & 1 & & Taubaté & 1 & & \\
\hline Itapeva & & 1 & & & Tietê & 1 & & \\
\hline Itapira & & 1 & 1 & & Torrinha & 2 & 1 & 1 \\
\hline Itapirapuã Paulista & & 1 & & & Tremembé & 1 & & \\
\hline Ituverava & & 1 & & & Ubatuba & 1 & & \\
\hline Jaboticabal & & & 1 & & Vargem Grande do Sul & 1 & 1 & 1 \\
\hline Jarinu & & 1 & & & Valent in Gentil & 1 & & \\
\hline Joanópolis & & 1 & & & Vista Alegre do Alto & 1 & & 1 \\
\hline Jundiai & & 3 & & & Votorantin & 1 & & \\
\hline
\end{tabular}


Os dados obtidos foram analisados estatisticamente, com exclusão, para a variável HMF, de dois dados, considerados discrepantes (outliers).

\section{RESULTADOS}

Os resultados obtidos para índice de diástase das amostras de méis produzidos no Estado de São Paulo encontram-se na Tabela 2.

Persono-Oddo et al. (1990) verificaram valores bastante variáveis para o índice de diástase de amostras de méis dentro da mesma florada (de 10 a 43 para méis de Castanea), o mesmo sendo verificado com as amostras analisadas na presente pesquisa, principalmente nas amostras de mel de flores silvestres cuja variação foi de 5,0 a 38,5.

A média do índice de diástase das amostras de méis de laranjeira foi significativamente menor $(9,4)$ do que a dos méis silvestre e de eucalipto (17,3 e 15,8, respectivamente) o que concorda com as observações de White et al. (1964), citado por Thrasyvoulou (1986).

Em outros trabalhos foram observados valores médios para o índice de diástase de: 20,12 $\pm 8,92$ (Martinez-Gomez et al., 1993); médias de 17,7 e 11,0, respectivamente, para as faixas de variação de 13,0 a 30,0 e 5,5 a 20,0 (Mesallam \& El-Shaarawy, 1987).

Bonvehí \& Coll (1995) observaram valores de 10,3 a 20 para índice de diastase em méis de citros da Espanha, Mendes et al. (1998) em amostras de méis portugueses verificaram valores de 8 a 22 e Al-Khalifa \& Al-Arify (1999), variações de 3,3 a 12 para méis da Arábia Saudita.

Balenovic et al. (1988) obtiveram 54,4\% das 44 amostras de méis analisadas em conformidade com os padrões oficiais e Vit-Oliver (1988) observou que $66 \%$ das amostras por ele estudadas estavam dentro das especificações do padrão venezuelano, valores estes muito inferiores aos $93,6 \%$, encontrados neste trabalho quando foram consideradas todas as amostras.

Segundo legislação brasileira, são tolerados índices de diástase de no mínimo 8, ou 3, se o valor do HMF for inferior a $15 \mathrm{mg} / \mathrm{kg}$. Dessa forma, 10 amostras não atingiram o valor mínimo exigido para índice de diastase (S-01; S-38; S-52; S-82; S-90 (5,3\% de amostras de méis de flores silvestres); E-09 (3,7\% de amostras de méis de flores de eucalipto); 
Tabela 2. Valores de índice de diástase das amostras de méis de flores silvestre, de eucalipto e de laranjeira coletadas em municípios do Estado de São Paulo, Brasil.

\begin{tabular}{|c|c|c|c|c|c|c|c|c|c|}
\hline \multicolumn{6}{|c|}{$\begin{array}{l}\text { Mel silvestre } \\
\text { (S) }\end{array}$} & \multicolumn{2}{|c|}{$\begin{array}{c}\text { Mel de } \\
\text { eucalipto (E) }\end{array}$} & \multicolumn{2}{|c|}{$\begin{array}{c}\text { Mel de } \\
\text { laranjeira }(L)\end{array}$} \\
\hline S-01 & 5,0 & S-35 & 13,9 & S-69 & 10,9 & E-01 & 17,9 & L-01 & 5,0 \\
\hline S-02 & 29,4 & S-36 & 6,5 & $S-70$ & 17,9 & E-02 & 23,8 & $\mathrm{~L}-02$ & 6,5 \\
\hline S-03 & 13,9 & S-37 & 17,9 & S-71 & 17,9 & E-03 & 10,9 & L-03 & 8,3 \\
\hline S-04 & 8,3 & S-38 & 5,0 & S-72 & 29,4 & E-04 & 17,9 & L-04 & 8,3 \\
\hline S-05 & 17,9 & S-39 & 13,9 & S-73 & 23,8 & E-05 & 13,9 & L-05 & 17,9 \\
\hline S-06 & 23,8 & $S-40$ & 10,9 & S-74 & 13,9 & E-06 & 13,9 & L-06 & 8,3 \\
\hline S-07 & 13,9 & $S-41$ & 6,5 & S-75 & 8,3 & E-07 & 10,9 & L-07 & 8,3 \\
\hline $\mathrm{S}-08$ & 8,3 & $\mathrm{~S}-42$ & 23,8 & S-76 & 17,9 & $\mathrm{E}-08$ & 6,5 & L-08 & 10,9 \\
\hline S-09 & 8,3 & $S-43$ & 38,5 & S-77 & 6,5 & E-09 & 5,0 & L-09 & 10,9 \\
\hline S-10 & 17,9 & $S-44$ & 17,9 & S-78 & 29,4 & E-10 & 10,9 & L-10 & 8,3 \\
\hline S-11 & 17,9 & $\mathrm{~S}-45$ & 13,9 & S-79 & 38,5 & E-11 & 13,9 & $\mathrm{~L}-11$ & 8,3 \\
\hline S-12 & 13,9 & $S-46$ & 17,9 & S-80 & 29,4 & E-12 & 10,9 & $\mathrm{~L}-12$ & 8,3 \\
\hline S-13 & 8,3 & $\mathrm{~S}-47$ & 10,9 & S-81 & 13,9 & E-13 & 23,8 & L-13 & 5,0 \\
\hline S-14 & 17,9 & S-48 & 13,9 & S-82 & 5,0 & E-14 & 17,9 & $\mathrm{~L}-14$ & 10,9 \\
\hline S-15 & 23,8 & $S-49$ & 13,9 & S-83 & 23,8 & E-15 & 17,9 & L-15 & 8,3 \\
\hline S-16 & 10,9 & S-50 & 29,4 & S-84 & 23,8 & E-16 & 23,8 & L-16 & 8,3 \\
\hline S-17 & 8,3 & S-51 & 38,5 & S-85 & 13,9 & E-17 & 13,9 & L-17 & 8,3 \\
\hline S-18 & 29,4 & S-52 & 5,0 & S-86 & 38,5 & E-18 & 13,9 & L-18 & $\mathbf{5 , 0}$ \\
\hline S-19 & 17,9 & S-53 & 38,5 & S-87 & 10,9 & E-19 & 23,8 & L-19 & 10,9 \\
\hline S-20 & 10,9 & S-54 & 17,9 & S-88 & 38,5 & E-20 & 10,9 & $\mathrm{~L}-20$ & 13,9 \\
\hline S-21 & 13,9 & S-55 & 8,3 & S-89 & 29,4 & $\mathrm{E}-21$ & 13,9 & L-21 & 13,9 \\
\hline S-22 & 13,9 & S-56 & 8,3 & S-90 & 5,0 & E-22 & 17,9 & $\mathrm{~L}-22$ & 10,9 \\
\hline S-23 & 8,3 & S-57 & 17,9 & S-91 & 10,9 & E-23 & 17,9 & L-23 & 10,9 \\
\hline S-24 & 17,9 & S-58 & 17,9 & S-92 & 17,9 & $\mathrm{E}-24$ & 23,8 & L-24 & 8,3 \\
\hline S-25 & 10,9 & S-59 & 6,5 & S-93 & 17,9 & E-25 & 23,8 & $\mathrm{~L}-25$ & 10,9 \\
\hline S-26 & 8,3 & $S-60$ & 23,8 & S-94 & 38,5 & $E-26$ & 17,9 & $\mathrm{~L}-26$ & 10,9 \\
\hline S-27 & 10,9 & S-61 & 17,9 & & & $\mathrm{E}-27$ & 8,3 & $\mathrm{~L}-27$ & 6,5 \\
\hline S-28 & 13,9 & S-62 & 29,4 & & & & & $\mathrm{~L}-28$ & 10,9 \\
\hline S-29 & 10,9 & $S-63$ & 13,9 & & & & & L-29 & 8,3 \\
\hline S-30 & 10,9 & S-64 & 29,4 & & & & & $\mathrm{~L}-30$ & 10,9 \\
\hline S-31 & 23,8 & S-65 & 17,9 & & & & & $\mathrm{~L}-31$ & 6,5 \\
\hline S-32 & 17,9 & $S-66$ & 17,9 & & & & & $\mathrm{~L}-32$ & 5,0 \\
\hline S-33 & 13,9 & S-67 & 23,8 & & & & & $\mathrm{~L}-33$ & 8,3 \\
\hline S-34 & 13,9 & S-68 & 13,9 & & & & & L-34 & 17,9 \\
\hline \multicolumn{3}{|c|}{ Número total de amostras } & \multicolumn{3}{|c|}{94} & \multirow{2}{*}{\multicolumn{2}{|c|}{$\begin{array}{c}15,77 \pm 1,08 \\
(1,17 \pm 0,18 a)\end{array}$}} & \multicolumn{2}{|c|}{34} \\
\hline \multicolumn{3}{|c|}{$\begin{array}{l}\text { Média } \pm s(\hat{m}) \\
\text { (dados transformados } \log x)\end{array}$} & \multicolumn{3}{|c|}{$\begin{array}{c}17,21 \pm 0,93 \\
(1,18 \pm 0,23 a)\end{array}$} & & & \multicolumn{2}{|c|}{$\begin{array}{c}9,41 \pm 0,54 \\
(0,95 \pm 0,14 b)\end{array}$} \\
\hline \multicolumn{3}{|c|}{$\begin{array}{l}\text { Intervalo de variação } \\
\text { CV }\end{array}$} & \multicolumn{3}{|c|}{5,0 a 38,5} & \multicolumn{2}{|c|}{5,0 a 23,8} & \multicolumn{2}{|c|}{5,0 a 17,9} \\
\hline
\end{tabular}

* Médias seguidas de mesma letra não diferem estatisticamente, pelo teste de Tukey, ao nível de $5 \%$ de probabilidade.

Em negrito- valores inferiores aos estabelecidos pela legislação vigente. 
L-01; L-13; L-18; L-32 (11,8\% de amostras de méis de flores de laranjeira).

No total das amostras, $6,4 \%$ não atingiram o valor mínimo do índice de diástase exigido pela legislação brasileira.

Os dados referentes ao HMF das 155 amostras de méis do Estado de São Paulo se encontram na Tabela 3.

O nível de HMF em méis recém-colhidos é muito baixo, aumentando significativamente por meio do armazenamento, aquecimento ou adição de açúcares invertidos (Doner, 1977, citado por Messallan \& El-Shaarawy, 1987). Desta forma, as amostras que não se enquadram nos limites estabelecidos podem ter sido submetidas a qualquer um dos processos citados.

Dentre as amostras não aceitáveis duas, S-90 e E-17, apresentaram valores discrepantes, 122,0 e $207,2 \mathrm{mg} / \mathrm{kg}$, evidenciando algum tipo de adulteração. Wootton \& Ryall (1985) por meio de dois métodos distintos obtiveram valores de HMF excessivamente altos para a mesma amostra, da ordem de 122 e $241 \mathrm{mg} / \mathrm{kg}$, comparáveis aos obtidos nas duas amostras citadas acima. Altos valores de $\operatorname{HMF}(120,0 ; 149,0 ; 160,0$ e 164,0 $\mathrm{mg} / \mathrm{kg}$ ) também foram observados por Messallan \& El-Shaarawy (1987).

Valores médios de 3,630 $\pm 2,553$ (Martinez-Gomez et al., 1993); 23,$0 ; 12,8 ; 15,2$ e $18,0 \mathrm{mg} / \mathrm{kg}$ (Tuveri \& Prosperi, 1985), também foram observados, sendo as últimas médias mais próximas às encontradas na presente pesquisa.

Marchini et al. (2000) analisaram características qualitativas de méis florais (de eucaliptos, silvestre e de laranjeira) que passaram por processo de descristalização (em microondas, banho-maria e sol) encontrando os seguintes valores médios de HMF para mel de flores silvestres: 11,$42 ; 15,36 ; 15,41 ; 15,50 ;$ mel de flores de laranjeira: 22,$08 ; 23,67$; 23,$57 ; 23,81$ e mel de flores de eucalipto: 22,$66 ; 24,05 ; 23,09 ; 23,28$.

Issa et al. (1998) constataram que méis escuros apresentaram valores de HMF maiores em relação aos méis mais claros. Amostras aquecidas a $65^{\circ} \mathrm{C}$ apresentaram um pequeno aumento de $\mathrm{HMF}$, mostrando que a análise deste parâmetro isoladamente não pode ser um indicador de qualidade.

A média dos valores de HMF para amostras de mel de eucalipto $(10,16)$ foi significativamente menor que a dos méis silvestre $\mathrm{e}$ de laranjeira (18,18 e 15,15, respectivamente). 
Tabela 3. Valores HMF $(\mathrm{mg} / \mathrm{kg}$ ) das amostras de méis de flores silvestre, de eucalipto e de laranjeira coletadas em municípios do Estado de São Paulo, Brasil.

\begin{tabular}{|c|c|c|c|c|c|c|c|c|c|}
\hline \multicolumn{6}{|c|}{$\begin{array}{l}\text { Mel silvestre } \\
\text { (S) }\end{array}$} & \multicolumn{2}{|c|}{$\begin{array}{c}\text { Mel de } \\
\text { eucalipto (E) }\end{array}$} & \multicolumn{2}{|c|}{$\begin{array}{c}\text { Mel de } \\
\text { laranjeira }(L)\end{array}$} \\
\hline $\mathrm{S}-01$ & 27,2 & S-35 & 10,3 & S-69 & 12,0 & E-01 & 20,0 & L-01 & 38,3 \\
\hline $\mathrm{S}-02$ & 8,1 & S-36 & 6,6 & S-70 & 28,4 & $\mathrm{E}-02$ & 12,6 & L-02 & 11,8 \\
\hline S-03 & 36,3 & S-37 & 3,4 & S-71 & 15,0 & E-03 & 16,3 & L-03 & 9,3 \\
\hline S-04 & 24,6 & S-38 & 20,0 & S-72 & 14,6 & E-04 & 5,8 & L-04 & 10,7 \\
\hline S-05 & 11,1 & S-39 & 12,8 & S-73 & 10,9 & E-05 & 15,1 & L-05 & 14,8 \\
\hline S-06 & 9,2 & $\mathrm{~S}-40$ & 15,3 & S-74 & 6,5 & E-06 & 5,0 & L-06 & 11,6 \\
\hline S-07 & 13,5 & $\mathrm{~S}-41$ & 11,8 & S-75 & 21,9 & E-07 & 5,7 & L-07 & 8,3 \\
\hline S-08 & 10,7 & $\mathrm{~S}-42$ & 11,1 & S-76 & 10,0 & E-08 & 11,9 & L-08 & 8,2 \\
\hline S-09 & 9,9 & $\mathrm{~S}-43$ & 11,5 & S-77 & 10,8 & E-09 & 27,9 & L-09 & 11,2 \\
\hline S-10 & 10,9 & $\mathrm{~S}-44$ & 4,9 & S-78 & 21,4 & E-10 & 12,0 & L-10 & 24,6 \\
\hline S-11 & 12,4 & $S-45$ & 22,7 & S-79 & 10,2 & E-11 & 5,9 & L-11 & 12,7 \\
\hline S-12 & 11,4 & $\mathrm{~S}-46$ & 19,1 & S-80 & 33,6 & E-12 & 19,9 & L-12 & 19,4 \\
\hline S-13 & 1,9 & $\mathrm{~S}-47$ & 14,2 & S-81 & 22,7 & E-13 & 5,4 & L-13 & 20,2 \\
\hline S-14 & 10,9 & $\mathrm{~S}-48$ & 20,2 & S-82 & 64,6 & E-14 & 2,0 & L-14 & 7,9 \\
\hline S-15 & 12,0 & $S-49$ & 34,2 & S-83 & 49,8 & E-15 & 2,2 & L-15 & 10,2 \\
\hline S-16 & 19,4 & S-50 & 4,9 & S-84 & 25,5 & E-16 & 2,0 & L-16 & 8,6 \\
\hline S-17 & 11,6 & S-51 & 1,0 & S-85 & 17,7 & E-17 & 207,2 & $\mathrm{~L}-17$ & 14,3 \\
\hline S-18 & 6,4 & S-52 & 15,2 & S-86 & 59,5 & E-18 & 10,7 & L-18 & 13,0 \\
\hline S-19 & 11,3 & S-53 & 19,9 & S-87 & 35,9 & E-19 & 9,1 & L-19 & 11,6 \\
\hline S-20 & 10,0 & S-54 & 18,0 & S-88 & 17,9 & E-20 & 36,1 & L-20 & 16,4 \\
\hline S-21 & 19,6 & S-55 & 19,5 & S-89 & 16,0 & E-21 & 6,0 & $\mathrm{~L}-21$ & 10,0 \\
\hline S-22 & 12,6 & S-56 & 15,4 & S-90 & 122,0 & $\mathrm{E}-22$ & 5,8 & $\mathrm{~L}-22$ & 15,4 \\
\hline S-23 & 9,7 & S-57 & 31,6 & S-91 & 17,9 & $E-23$ & 8,1 & $\mathrm{~L}-23$ & 19,8 \\
\hline S-24 & 10,9 & S-58 & 14,4 & S-92 & 16,8 & E-24 & 6,4 & L-24 & 16,5 \\
\hline S-25 & 19,5 & S-59 & 6,9 & S-93 & 19,1 & E-25 & 0,3 & L-25 & 10,2 \\
\hline S-26 & 4,1 & S-60 & 12,7 & S-94 & 25,7 & E-26 & 1,5 & L-26 & 15,7 \\
\hline S-27 & 24,9 & S-61 & 18,9 & & & E-27 & 10,5 & L-27 & 13,1 \\
\hline S-28 & 23,6 & S-62 & 36,9 & & & & & L-28 & 15,6 \\
\hline S-29 & 17,8 & $S-63$ & $\mathbf{5 8 , 9}$ & & & & & L-29 & 13,6 \\
\hline $\mathrm{S}-30$ & 10,2 & S-64 & 6,1 & & & & & L-30 & 15,4 \\
\hline S-31 & 19,3 & S-65 & 11,3 & & & & & $\mathrm{~L}-31$ & 13,9 \\
\hline S-32 & 20,2 & S-66 & 17,0 & & & & & L-32 & 45,3 \\
\hline S-33 & 59,5 & S-67 & 33,7 & & & & & L-33 & 13,9 \\
\hline S-34 & 13,7 & S-68 & 11,5 & & & & & L-34 & 13,7 \\
\hline \multicolumn{4}{|c|}{ Número total de am ostras analisadas } & \multicolumn{2}{|c|}{93} & & & \multicolumn{2}{|c|}{34} \\
\hline \multicolumn{4}{|c|}{$\begin{array}{l}\text { Média } \pm s(m) \\
\text { (dados transformados em } x^{-0,3} \text { ) }\end{array}$} & \multicolumn{2}{|c|}{$\begin{array}{c}18,18 \pm 1,29 \\
(2,29 \pm 0,45 a)\end{array}$} & \multicolumn{2}{|c|}{$\begin{array}{c}10,16 \pm 1,66 \\
(1,86 \pm 0,51 b)\end{array}$} & \multicolumn{2}{|c|}{$\begin{array}{c}15,15 \pm 1,33 \\
(2,22 \pm 0,28 a)\end{array}$} \\
\hline \multicolumn{4}{|c|}{ Intervalo de variação } & \multicolumn{2}{|c|}{1,0 a 64,6} & \multicolumn{2}{|c|}{0,3 a 36,1} & \multicolumn{2}{|c|}{7,9 a 45,3} \\
\hline \multicolumn{4}{|c|}{ Dados excluídos } & \multicolumn{2}{|c|}{122,0} & \multicolumn{2}{|c|}{$\begin{array}{r}207,0 \\
1946 \%\end{array}$} & \multirow{2}{*}{\multicolumn{2}{|c|}{-}} \\
\hline $\mathrm{CV}$ & & & & & & 19, & & & \\
\hline
\end{tabular}

* Médias seguidas de mesma letra não diferem estatisticamente, pelo teste de Tukey, ao nivel de $5 \%$ de probabilidade.

Em negrito- valores superiores aos estabelecidos pela legislação vigente. 
Vários autores como Thrasyvoulou et al. (1982), Colin et al. (1986), Balenovic et al. (1988), Vit-Oliver (1988) e Bricage (1989) verificaram porcentagens das amostras de méis analisados que estavam dentro dos limites, para HMF, estabelecidos pela legislação do país, que variaram de apenas 18 até $88 \%$.

Piazza \& Accorti (1983) observaram 100\% das amostras de méis produzidos por pequenos apicultores, $97 \%$ das amostras adquiridas em cooperativas, $76 \%$ das adquiridas no comércio e $55 \%$ das importadas pela Itália, dentro dos limites estabelecidos pela legislação européia.

No presente trabalho verificou-se que $94,8 \%$ do total das amostras estudadas apresentaram valores de HMF dentro dos limites estabelecidos pela legislação brasileira, sendo consideradas não aceitáveis apenas 8 amostras (S-33; S-63; S-82; S-83; S-86; S-90 (6,2\% de amostras de méis de flores silvestres); E-17 (3,7\% de amostras de méis de flores de eucalipto)e L-32 (2,9\% de amostras de méis de flores de laranjeira)).

\section{CONCLUSÕES}

Conclui-se que a caracterização das amostras estudadas no presente trabalho é estabelecida por faixas de variação de valores obtidos de 5,0 a 38,5 para o índice de diástase e 0,3 a $242,6 \mathrm{mg} / \mathrm{kg}$ para HMF. Das amostras analisadas, 93,6\% se enquadram nas especificações da legislação brasileira para qualidade de mel para índice de diástase e 94,8\% para HMF.

\section{REFERÊNCIAS BIBLIOGRÁFICAS}

AL-KHALIFA, A.S.; I.A. AL-ARIFY, 1999. Physicochemical characteristics and pollen spectrum of some Saudi honeys. Food Chemistry, v. 67, n.1, p.21-25.

AZEREDO, M.A.A.; L.C. AZEREDO, 1999. Características físico-químicas dos méis do município de São Fidelis, RJ. Ciência e Tecnologia de Alimentos, v.19, n.1, p.3-7.

BALENOVIC, J.; M. PERKOVAC, J. PAPIC, 1992. Hidroxymethylfurfural content and amylase activity in honey. 
Prehrambeno Tehnoloska Revija, v.26, n.4, p.143-146, 1988. Resumo em CAB Abstracts on CD-ROM, v.3B.

BONVEHÍ, J.S.; F.V. COLL, 1995. Characterization of citrus honey

(Citrus spp.) produced in Spain. Journal of Agricultural Food Chemistes, v. 43, p.2053-2057,

BRICAGE, P., 1990-91. The amount of HMF in honeys does it have an effect on the health of the consumer? Bulletin Technique Apicole, v.16, n.4, p. 255-262,1989. Resumo em CAB Abstracts on CD-ROM, v.3A.

COLIN, M.E.; C. FLAMINI; J. MALAUSSENE; J. POURTALIER, J., 1987-89. The quality of commercial honeys. Cahiers de Nutrition et de Dietetique, v. 21, n.3, p. 219-222, 1986. Resumo em CAB Abstracts on CD-ROM, v.2.

ISSA, M.R., D. JONG; H. HORN, 1988. Características físico-químicas de amostras de méis normais, aquecidos e misturados. In: CONGRESSO BRASILEIRO DE APICULTURA, 12, Salvador, 1998. Anais. Salvador: Confederação Brasileira de Apicultura, p.209.

MARCHINI, L.C.; A.C.L. RODRIGUES, C.C.C. MORETI, 2000. HMF (Hidroximetilfurfural) e diastase de méis submetidos à dissolução de cristais por aquecimento. Boletim de Indústria animal, v. 57, n.1, p.85-91.

MARTINEZ-GOMEZ, M.E.; E. GUERRA HERNANDEZ; J.Y. MONTILLA GOMEZ; J.L MOLINS MARIN, 1993. Physicochemical analysis of Spanish commercial Eucalyptus honeys. Journal of Apicultural Research, v.32, n.3/4, p. 121-126.

MENDES, E.; E.B. POENÇA; I.M.P.L.V.O. FERREIRA, et al., 1998. Quality evaluation of portuguese honey. Carbohydrate Polymers, v.37, n.3, p.219-223.

MESALLAN, A S.; M.I. EL-SHAARAWY, 1987. Quality attributes of honey in Saudi Arabia. Food Chemistry, v. 25, n.1, p.1-11.

PERSONO-ODDO, L.; E. VALDI, M. ACCORTI, 1990. Diastatic activity in some unifloral honeys. Apidologie, v.21, n.1, p. 17-24.

PIAZZA, M.G.; M. ACCORTI, 1984-86. HMF of domestic and foreign honeys sold in Italy. Apitalia, v.10. n.1., p.8-10, 1983. Resumo em 